\title{
USING NETWORK ANALYSIS TO EXPLORE COGNITIVE DOMAINS IN PATIENTS WITH UNIPOLAR VERSUS BIPOLAR DEPRESSION: A PROSPECTIVE NATURALISTIC STUDY
}

- Cesare Galimberti ${ }^{1}, \mathrm{MD}$, Psychiatrist

- Monica Francesca Bosi², MD, Psychiatrist, Chief of the Centre for the Treatment of Depressive Disorders, Unit of Psychiatry 2, ASST Fatebenefratelli-Sacco, Milan, Italy

- Valentina Caricasole ${ }^{2}, \mathrm{MD}$, Resident in Psychiatry at University of Milan

- Riccardo Zanello ${ }^{2}, \mathrm{MD}$, Resident in Psychiatry at University of Milan

- Bernardo Dell'Osso 2,3,4, MD, Associate Professor of Psychiatry at University of Milan, Director of the Unit of Psychiatry 2, ASST Fatebenefratelli-Sacco, Milan, Italy

- Caterina Adele Vigano ${ }^{2 *}, \mathrm{MD}, \mathrm{PhD}$, Assistant Professor of Psychiatry at University of Milan

${ }^{1}$ Department of Mental Health, ASST Rhodense, Garbagnate Milanese, Milan, Italy 2Psychiatry Unit, Department of Biomedical and Clinical Sciences "Luigi Sacco", University of Milan, ASST Fatebenefratelli-Sacco, Milan, Italy.

${ }^{3}$ Department of Psychiatry and Behavioural Sciences, Bipolar Disorders Clinic, Stanford University, CA, USA.

${ }^{4} \mathrm{CRC}$ "Aldo Ravelli" for Neurotechnology and Experimental Brain Therapeutics, University of Milan, Milan, Italy.

\section{*Corresponding author:}

Caterina Viganò, M.D., PhD

Assistant Professor of Psychiatry

University of Milan, Department of Biomedical and Clinical Sciences "Luigi Sacco" ASST Fatebenefratelli-Sacco, via G.B. Grassi 74, 20157 Milan, Italy.

Phone +390239042904

e-mail: caterina.vigano@unimi.it 


\section{ABSTRACT}

Objective: Despite growing evidence in the field of cognitive function in mood disorders, the neurocognitive profiles of patients with unipolar and bipolar depression still need further characterization. In this study, we applied network analysis, hypothesizing this approach could highlight differences between Major Depressive Disorder (MDD) and Bipolar Disorder (BD) from a cognitive perspective.

Methods: The cognitive performance of 109 patients (72 unipolar and 37 bipolar depressed outpatients) was assessed through the Montreal Cognitive Assessment (MoCA) and a series of clinical variables was collected. Differences in cognitive performance between MDD and BD patients were tested using non parametric tests. Moreover, a network graph representing MoCA domains as nodes and Spearman's rho correlation coefficients between the domains as edges was constructed for each group.

Results: The presence of mild cognitive impairment was observed both in MDD and BD patients during depression. No statistical significant difference was found between the two groups in terms of overall cognitive performance and across single domains. Nonetheless, network analytic metrics demonstrated different roles of memory and executive dysfunction in MDD vs BD patients: in particular, MDD network was more densely interconnected than BD network and memory was the node with the highest betweenness and closeness centrality in MDD, while executive function was more central in $\mathrm{BD}$.

Conclusions: From a network analytic perspective, memory impairment displays a central role in the cognitive impairment of patients with unipolar depression, whereas executive dysfunction appears to be more central in bipolar depression. Further research is warranted to confirm our results.

Keywords: cognitive impairment, network analysis, bipolar disorder, major depressive disorder, acute depression, remission.

\section{Introduction}

Cognitive impairment is one of the most important psychopathological features in both major depressive disorder (MDD) and bipolar disorder (BD), particularly during major depressive episodes (MDE). Over the last few decades, the number of studies addressing neurocognitive functioning in mood disorders has grown exponentially ${ }^{1}$, with increasing scientific interest about the influence of cognitive impairment on the course of these disorders ${ }^{2,3}$, determining a more severe impairment of global functioning and a higher degree of overall disability ${ }^{4-8}$. Furthermore, cognitive impairment is currently considered a crucial therapeutic target in depression, in order to achieve cognitive remission ${ }^{9}$ and recovery ${ }^{10}$.

From a cognitive perspective, many studies have demonstrated that depressed patients exhibit a wide range of cognitive deficits both in MDD and BD. These deficits do not necessarily depend on the mood state ${ }^{11,12}$ and mainly regard the domains of memory ${ }^{13-18}$, attention ${ }^{14,19-21}$, executive functions ${ }^{22-25}$ and language ${ }^{26}$.

However, few studies have directly compared the cognitive profiles of patients with MDD and $\mathrm{BD}^{18}$. In addition, these studies included patients regardless of illness phase and produced conflicting evidence, showing, in some cases, different cognitive alteration profiles between unipolar and bipolar patients (both in terms of expression and severity), and overlapping deficits in other $\operatorname{cases}^{27}$. For instance, in a study comparing three groups of patients with MDD, BD-I and BDII, Xu and colleagues (2012) found similar patterns of cognitive dysfunction during acute depression across the 3 groups, but observed a globally greater cognitive impairment in BD-I 
compared to BD-II and MDD ${ }^{27}$. Daniel and co-workers (2013) confirmed that both patients with MDD and BD-I showed some cognitive deficits in relation to information processing speed compared to healthy controls, but they could not find any specific difference between the two subtypes of mood disorder ${ }^{28}$. Moreover, overlapping alterations in bipolar and unipolar patients were observed by Hermens et al. (2010) ${ }^{29}$. Finally, while some authors have shown greater deficiency in executive functions in bipolar versus unipolar patients ${ }^{30}$, at least one study has not shown significant differences in this domain ${ }^{31}$. Indeed, in literature there is also evidence for a higher degree of cognitive impairment in unipolar compared to bipolar depression, regarding memory, executive functions and decision-making ${ }^{32}$, as well as executive functions and psychomotor speed in drug-naive patients ${ }^{33}$.

Based on the above, to date, the presence of distinctive profiles between unipolar and bipolar depression from a cognitive perspective is still debated. In clinical settings, however, that would add useful elements to the differential diagnosis between these two nosographic entities, which are often confused and misdiagnosed ${ }^{34}$. Currently, the elements considered suggestive of bipolarity during depression are mainly represented by psychopathological symptoms and clinical variables (such as the presence of irritability, psychomotor agitation, emotional lability, rapid thoughts, psychotic symptoms, atypical depressive symptoms including hypersomnia and hyperphagia) ${ }^{34,35}$, but do not comprise any cognitive specifier.

The limited evidence about the distinctive characteristics of cognition in MDD and BD may actually reflect the presence of methodological biases of the aforementioned studies (above all, the enrollment of patients during different phases of illness) and the lack of sensitivity of classical statistical methods in the analysis of potential differences.

For these reasons, in the present study, the problem of the differential diagnosis between the cognitive profiles of unipolar and bipolar depression was addressed by conducting a network analysis, an innovative analytical model that is gaining growing interest in the study of psychopathology. Thus, our main objective was to evaluate the differences between the cognitive profiles of patients with MDD and BD during depression, by performing a network analysis of the cognitive domains of the Montreal Cognitive Assessment (MoCA) ${ }^{36}$. A secondary objective was to confirm the usefulness of the MoCA as a cognitive screening tool in depression, and then after remission, by estimating the rate and severity of cognitive impairment in a naturalistic sample of depressed outpatients.

\section{Methods}

Eligibility for the study

The present study had a prospective, observational design and it was conducted in a naturalistic setting, namely the Centre for the Diagnosis and Treatment of Depressive Disorders (CTDD) of the ASST Fatebenefratelli-Sacco in Milan, an outpatient, tertiary psychiatric service dedicated to the treatment of affective disorders. Both outpatients and patients undergoing day-hospital care between January 2016 and December 2017 were considered eligible.

The design of this study fitted most of the methodological criteria defined by the International Society for Bipolar Disorders (ISBD) task force, as stated in the guidelines for the study of cognitive disorders in $\mathrm{BD}^{37}$, as well as those previously proposed by Burdick and colleagues $(2015)^{38}$, although it was conceived before their publication.

Both subjects of male and female gender, above the age of 18, with a current diagnosis of MDE within MDD, BD-I or BD-II were considered eligible for the study. Diagnoses were formulated through a structured clinical interview complying with the criteria of the Diagnostic and Statistical Manual of Mental Disorders-Fifth Edition (DSM-5) ${ }^{39}$. Inclusion and exclusion criteria are summarized in table 1. 
The main demographic and clinical characteristics of the sample were collected in an electronic database through a retrospective chart review, both in traditional records and computerized form or, when necessary, through a direct interview with the subject enrolled in the study.

All subjects enrolled in the study provided informed consent to the collection of personal and sensitive data, as well as the consent to undergo the psychometric evaluation and cognitive test described below.

\section{Psychopathological assessment}

The severity of depressive symptomatology was assessed with the 21 items-Hamilton Depression Rating Scale (HDRS) ${ }^{40}$ at the time of entry into the study (depressive phase) and at clinical remission (remission, defined as a HDRS total score of less than 8 according to literature). In addition, at the time of entry into the study (depression) and at remission (remission), the severity of the anxious component was assessed with the Hamilton Anxiety Rating Scale (HARS) ${ }^{41}$. Clinical remission was considered complete upon the achievement of a concomitant score of HDRS $<8$ and HARS $<7$.

Cognitive screening: the Montreal Cognitive Assessment (MoCA)

In this study, cognitive performance was assessed with the Montreal Cognitive Assessment (MoCA), administered during the depressive phase and after clinical remission (as defined above). The MoCA is a short screening tool developed by Nasreddine et al. (2005) for the identification of mild cognitive impairment (MCI), a clinical condition of increased risk for the subsequent development of Alzheimer's disease or other forms of dementia ${ }^{36}$.

The MoCA examines six different cognitive domains: executive functions, visuospatial abilities, attention, verbal fluency, memory and spatio-temporal orientation. Through the measurement of different cognitive domains, the total score of the MoCA, which varies from a minimum of 0 to a maximum of 30, provides a global assessment of the cognitive performance of the subject. In relation to the years of educations, a score equal to or above 26 is considered normal ${ }^{36}$, and a supplementary point is added if years of education are less than 12 . The average time to administer the test is usually around 10-15 minutes.

The MoCA has good internal consistency with a Cronbach's alpha value of $0.83^{36}$. Furthermore, the sensitivity and the specificity of the MoCA in identifying MCI are $90 \%$ and $87 \%$, respectively. Finally, the positive (VPP) and negative (VPN) predictive value of the MoCA are $89 \%$ and $91 \%$ for MCI [40].

Among cognitive screening tests, the Mini Mental State Examination (MMSE) ${ }^{42}$ is the most commonly used for the detection of cognitive impairment. However, the MMSE is considered less sensitive in detecting deficits in executive functions, attention and visuospatial domains ${ }^{36,43}$. Compared to the MMSE, the MoCA includes several tasks specifically aimed at evaluating executive functions, attention ${ }^{44}$ and memory.

The MoCA has proven to be a sensitive tool for screening patients with other forms of dementia, like, for instance, vascular dementia ${ }^{45}$, dementia associated with Parkinson's disease ${ }^{46}$ and finally Frontotemporal Dementia ${ }^{47}$. With respect to psychiatric disorders, the MoCA has been used successfully to evaluate cognitive impairment in patients with depression, BD and schizophrenia ${ }^{48,49}$ as well as in depressed patients undergoing electroconvulsive therapy (ECT) ${ }^{50}$.

\section{Statistical analysis}

The Kolmogorov-Smirnov and Shapiro-Wilk tests were performed to test the assumption of normal distribution of the clinical and sociodemographic variables, as well as that of HDRS, HARS and MoCA scores. 
In MDD, with the exception of age, $\mathrm{D}(61)=0.074, \mathrm{p}=0.200$, HDRS score in the depressive phase, $\mathrm{D}(61)=0.078, \mathrm{p}=0.200$, and HARS score in the depressive phase, $\mathrm{D}(61)=0.071, \mathrm{p}=0.200$, data regarding all other variables violated the assumption of normal distribution.

In $\mathrm{BD}$, a normal distribution was found for age, $\mathrm{D}(33)=0.100, p=0.200$, age of onset, $\mathrm{D}(33)=0.111$, $\mathrm{p}=0.200$, MoCA score in depressive phase, $\mathrm{D}(33)=0.142, \mathrm{p}=0.087$, HDRS score in the depressive phase, $\mathrm{D}(33)=0.090, \mathrm{p}=0.200$, HARS score in the depressive phase, $\mathrm{D}(33)=0.127, \mathrm{p}=0.195$, and HDRS score in clinical remission phase, $\mathrm{D}(33)=0.128, \mathrm{p}=0.186$. Data regarding all other sociodemographic and clinical variables violated the assumption of normal distribution.. Thus, based on these preliminary analyses, non-parametric tests were used for the statistical analyses described below.

Demographic and clinical characteristics were compared between the two groups using MannWhitney $U$ non-parametric test for continuous variables and Chi-square $\left(\chi^{2}\right)$ test for dichotomous variables.

Then, Mann-Whitney $U$ test was used to analyse the differences between the scores of the single cognitive domains of the MoCA between the two groups and between the total scores of the scales for depression and anxiety. These analyses were conducted for both the depressive and remissionrelated scores. Furthermore, the statistical significance of the change of the MoCA total scores and of the individual cognitive domains from the depressive phase to clinical remission was calculated using the Wilcoxon signed-rank test.

For the two groups of patients with MDD and BD, the matrix of Spearman rho $(\rho)$ correlation coefficients between the six MoCA domains (memory, executive functions, verbal fluency, orientation, attention, visuospatial abilities) was calculated.

In addition, for each of the two groups examined, we constructed a network graph in which the nodes represent the six cognitive domains of the MoCA and the edges represent Spearman's rho correlation coefficients between the domains. The cognitive networks for unipolar and bipolar depression were thus constructed. Within both networks, only the connections corresponding to a coefficient $\rho \geq 0.20$ were represented. Furthermore, in order to allow an immediate graphical comparison, the nodes within the networks have been arranged using a circular layout.

Finally, through a network analysis, for each network we calculated three metrics defined as follows:

- Network density: corresponding to the average of the correlation coefficients, network density expresses the degree of intercorrelation between the symptoms of a network, and therefore their tendency to present themselves simultaneously ${ }^{51}$;

- Closeness centrality: a parameter indicating how easily all the other nodes of a network can be reached starting from the node examined, that is the average distance of that node from all the others. The nodes with the highest closeness quickly affect the other nodes and they are more influenced by the other nodes ${ }^{52,53}$;

- Betweenness centrality: it expresses the number of times a node is involved in the shortest path length between two other nodes. The nodes with a high betweenness are those that facilitate connections within a network ${ }^{52,53}$.

For all the analyses, the level of statistical significance was defined as $\mathrm{p}<0.05$. Statistical analyses were performed using IBM SPSS version 22.0, while network analysis and the construction of the network graph were obtained using $\mathrm{R}^{54}$ and Cytoscape version 3.2.155.

\section{Results}

Sample characteristics

A total of 109 outpatients with a MDE was enrolled in the study: 72 patients $(66.1 \%)$ diagnosed with MDD and 37 patients (33.9\%) diagnosed with BD. The two groups of patients were homogeneous with regard to most of the main demographic and clinical variables (see table 2 ). The two groups significantly differed with regard to previous hospitalizations $\left(\chi^{2}=14.924, \mathrm{p}<0.001\right)$, previous suicide attempts $\left(\chi^{2}=4.688, p=0.030\right)$ and number of mood episodes $(U=566.0, Z=-4.028$, $\mathrm{p}<0.001)$. Moreover, some differences emerged in terms of current medication status, as patients 
with BD were more frequently treated with antipsychotics $\left(\chi^{2}=5.541, \mathrm{p}=0.019\right)$ and mood stabilizers $\left(\chi^{2}=23.096, p<0.001\right)$, but less frequently with antidepressants $\left(\chi^{2}=8.224, p=0.004\right)$ compared to patients with MDD. However, there was no difference in treatment with benzodiazepines $\left(\chi^{2}=1.097\right.$, $\mathrm{p}=0.295$ ). The aforementioned differences likely reflected the naturalistic context in which the study was conducted because, in clinical practice, MDD and BD are characterized by a different course of illness and by different treatment strategies. We took into consideration these differences by conducting some analyses to weight their impact on the variables examined in the present study. During depression, mean HDRS score and mean HARS were respectively $27.35 \pm 6.05$ and $21.61 \pm 9.062$ for MDD, and $26.62 \pm 6.45$ and $18.68 \pm 7.32$ for BD. Therefore, the study sample presented with moderate to severe depressive symptomatology and with mild to moderate anxious symptomatology, as expected from the recruitment in a day-hospital service that is specifically dedicated to the treatment of severe forms of depression. No statistically significant differences emerged between the two groups with respect to depressive symptomatology ( $U=1265.5, Z=-0.311$, $\mathrm{p}=0.756)$ nor with respect to anxious symptomatology $(\mathrm{U}=1054.5, \mathrm{Z}=-1.557, \mathrm{p}=0.115)$.

\section{Cognitive performance during depression}

The mean MoCA total score (adjusted for education level) in the entire sample was 24.04 3.94 , lower than the normality cut-off of 26 , as defined by the normative studies of the test. Globally, $55.9 \%$ of the subjects enrolled for the study had mild cognitive impairment, defined as a MoCA score of less than 26. In the two groups, $54.2 \%$ of patients with MDD and $59.4 \%$ of patients with BD had cognitive impairment, with a mean MoCA score of $24.11 \pm 4.04$ and $23.89 \pm 3.78$ respectively, and no statistically significant difference between them $(\mathrm{U}=1274.0, \mathrm{Z}=-0.373$, $\mathrm{p}=0.709$ ).

Analysing the single cognitive domains of the MoCA, the total score of the memory domain was respectively $2.35 \pm 1.71$ and $2.32 \pm 1.58$ for MDD and $\mathrm{BD}$, with no statistically significant differences between the two groups $(\mathrm{U}=1313.0, \mathrm{Z}=-0.124, \mathrm{p}=0.902)$.

During depression, no statistically significant difference emerged between unipolar and bipolar patients, even considering single domains, as shown in table 3.

\section{Cognitive performance after clinical remission}

On average, MDD and BD patients achieved remission after 77.43 \pm 34.85 days and $84.32 \pm 37.84$ days respectively, with no statsstically significant difference between the two groups ( $\mathrm{U}=1195.5$, $\mathrm{Z}=-0.875$, $\mathrm{p}=0.382$ ). At time of clinical remission, the mean MoCA total score in the sample was $25.94 \pm 2.60$. In patients diagnosed with MDD the mean score was $25.81 \pm 2.65$, while it was $26.22 \pm 2.50$ in patients with BD. Therefore, once remitted, patients with MDD remained on average below MoCA threshold of normality, whereas patients with BD achieved normal levels. Anyway, this difference was not statistically significant $(\mathrm{U}=1188.0, \mathrm{Z}=-0.930, \mathrm{p}=0.353)$. Analysing the single cognitive domains of the MoCA, there were no statistically significant differences between the two groups in any cognitive domain: Memory $(\mathrm{U}=1301.0, \mathrm{Z}=-0.206, \mathrm{p}=0.837)$, Visuospatial skills $(\mathrm{U}=1241.5, \mathrm{Z}=-0.608, \mathrm{p}=0.543)$, Executive Functions $(\mathrm{U}=1249.5, \mathrm{Z}=-0.611, \mathrm{p}=0.541)$, Attention $(\mathrm{U}=1319.0, \mathrm{Z}=-0.115, \mathrm{p}=0.908)$, Verbal Fluency $(\mathrm{U}=1255.5, \mathrm{Z}=-0.540, \mathrm{p}=0.589)$, Orientation $(\mathrm{U}=1250.0, \mathrm{Z}=-0.855, \mathrm{p}=0.393)$.

Change in cognitive performance between depression and remission

All subjects enrolled in the study showed improvement in overall cognitive performance between depression and remission. There was indeed a statistically significant change in MoCA score both in the group of patients with $\operatorname{MDD}(Z=-7.326, p<0.001)$ and in the group of patients with BD $(\mathrm{Z}=3.773, \mathrm{p}<0.001)$.

Taking into consideration the single MoCA domains separately, in MDD patients there was a statistically significant improvement in the domains of Memory $(Z=-4.273, p<0.001)$, Executive 
Functions $(\mathrm{Z}=-3.431, \mathrm{p}=0.001)$ and Attention $(\mathrm{Z}=-2.389$, $\mathrm{p}=0.017)$, while there was no statistically significant variation in the domains of Visuospatial Abilities $(Z=-0.576, p=0.564)$, Verbal Fluency $(\mathrm{Z}=-1.531, \mathrm{p}=0.126)$ and Orientation $(\mathrm{Z}=-0.401, \mathrm{p}=0.688)$.

Likewise, in BD, there was a statistically significant variation in Memory $(Z=-3.559, p<0.001)$, Executive Functions $(\mathrm{Z}=-3.431, \mathrm{p}=0.001)$ and Attention $(\mathrm{Z}=-2.234, \mathrm{p}=0.020)$, while no significant changes were found in any other domain: Visuospatial Abilities $(Z=-0.530, p=0.596)$, Verbal Fluency $(\mathrm{Z}=-1.554, \mathrm{p}=0.120)$ and Orientation $(\mathrm{Z}=-1.734, \mathrm{p}=0.083)$.

Correlations between MoCA domains

We calculated the matrix of Spearman's rho $(\rho)$ correlation coefficients (given the non-parametric distribution of the variables) among the scores of the six MoCA domains, both for MDD and for BD patients (see table 5).

In patients with unipolar depression, 11 of the 14 correlation coefficients between cognitive domains were statistically significant. In the bipolar group, a lower proportion of statistically significant correlations emerged compared to unipolar patients (8 out of 15). After transforming MoCA single domains score into $z$ scores and calculating Spearman's $\rho$ correlations between them, no statistical significant differences emerged between the single domains correlations in MDD and BD patients.

Network analysis of MoCA domains

As described in the "Methods" section, the matrix of intercorrelations between the domains of the MoCA was used to construct a network graph for unipolar depression and one for bipolar depression. The two graphs are shown in Figures 1 and 2: the six nodes represent the domains of the MoCA, while the edges express the correlation existing between the two domains they connect. The higher the correlation value the thicker the line connecting the nodes. Within the networks, only moderate to strong correlations were expressed $(\rho>0.20)$.

The number of edges differed between the two networks: in fact, there were 13 edges in the network of unipolar depression and 10 in that of bipolar depression.

As previously described, three statistical parameters were calculated for each of the two networks: | density, betweenness centrality and closeness centrality. The so-called "network density" is mathematically corresponding to the average of the correlation coefficients between the domains.

High density can be clinically interpreted as the patients' tendency to have homogeneous patterns of cognitive impairment, affecting multiple domains at the same time, because if the density is higher, a patient developing deficits in one single domain is more likely to develop deficits in the other domains. In our study, the network of patients with unipolar depression had a higher density ( $\rho=0.34 \pm 0.13 ; 95 \%$ CI:0.26-0.41) than the network of patients with bipolar depression $(\rho=0.29 \pm 0.12 ; 95 \%$ CI: $0.17-0.41)$.

In Table 5, cognitive domains with the highest closeness centrality and betweenness centrality are reported. These two metrics can be calculated for each node of the network and, with different meanings, they express the specific role of that node within the network of symptoms.

As described in the section on statistical analysis, closeness centrality is a parameter that expresses | the ease with which all the other nodes of a network can be reached from the node examined, that is the average distance of that node from all the others. The betweenness centrality, on the other hand, expresses the number of times a node is involved in the shortest passage (or shortest path length) between two other nodes. The nodes with a high betweenness are those that facilitate connections within the network, while those with the highest closeness affect the other nodes faster and they are in turn more influenced by the other nodes ${ }^{52,53}$.

In the unipolar depression network, the domain with the highest closeness and betweenness was Memory, followed by the domains of Executive Functions and Attention, respectively.

On the other hand, in the network of bipolar depression, the domain of Executive Functions was the one with the highest closeness and betweenness, followed by the Attention. Verbal fluency was the third domain for betweenness, while Memory and Visuospatial Skills were third in closeness. 


\section{Discussion}

To our knowledge, this is the first study investigating the distinctive features of unipolar and bipolar depression from a cognitive perspective, through the application of an innovative statistical approach, such as the network analysis. Until now, research implementing network analysis in psychiatry has mainly focused on the interaction between psychopathological symptoms in mood and anxiety disorders ${ }^{56}$, and more recently on schizophrenia ${ }^{52}$. To date, there are no studies in literature that have applied network analysis to the investigation of cognitive impairment in depression.

Consistently with available literature, the results of our study confirmed that patients suffering from depression, regardless of longitudinal diagnosis, experience mild cognitive impairment. In fact, in our sample, more than 1 out of 2 depressed patients presented with cognitive impairment.

Confirming the usefulness of MoCA as a screening measure in depression, our result was consistent with epidemiological studies reporting a prevalence of cognitive impairment in MDD around 25$50 \% 57$, even though the real prevalence of cognitive alterations in depression is not fully clarified yet ${ }^{58,59}$. In a recent publication, Douglas et al. (2018) found variable prevalences of $14.7-52.9 \%$ and 32.2-64.4\% in outpatients with MDD and BD, respectively, in the depressive phase depending on the applied definition of cognitive impairment ${ }^{58}$, and therefore substantially consistent with our epidemiological finding.

The main objective of our study was to highlight the distinctive features of unipolar and bipolar depression from a cognitive perspective, as to date evidence on this topic is scarce and contrasting.

Using classical statistical techniques, we found overlapping patterns of cognitive impairment between patients with MDD and BD, quantitatively in terms of severity and qualitatively in terms of profile of involved domains. This result is consistent with what has been previously reported by several authors: Bearden and colleagues $(2006)^{18}$, for instance, showed similar patterns of cognitive deficits in unipolar and bipolar patients, which did not appear to depend on the clinical status but rather related to a common pathophysiological genesis, regarding temporal lobe dysfunction; Hermens and co-workers (2010), analyzing the neurocognitive profile of young unipolar and bipolar patients, observed overlapping alterations primarily linked to verbal memory impairment ${ }^{15}$; moreover, Daniel et al. (2013) had shown that performance on neurocognitive tests did not differentiate patients affected by MDD from those suffering from BD I ${ }^{28}$. Finally, similar neurocognitive alteration patterns have also been reported by Xu et al. (2012) 27 and, previously, by Sweeney and co-authors $(2000)^{20}$.

One strength of the present study lies in the enrollment of unipolar and bipolar patients during the acute depressive phase, whereas the samples of the aforementioned studies were heterogeneous and constituted by patients in different phases of illness (i.e., depressive/manic and euthymic).

Furthermore, compared to the present study, most of the mentioned studies were conducted on smaller samples.

Of note, cognitive assessment is generally obtained through the administration of extensive batteries of neuropsychological tests, while we characterized it by implementing a cognitive screening test of quick and easy administration. Although neuropsychological tests may be preferable in order to perform a more comprehensive analysis of a patient's cognitive deficits, in our opinion, the results of the present study confirm the usefulness of the MoCA as a screening tool in everyday clinical practice, avoiding the risk of confining cognitive assessment solely to research contexts.

With reference to the main objective of our study, the application of network analysis provided additional information, compared to what has been produced with traditional statistical methods, on the differential cognitive alterations characteristic of unipolar and bipolar depressed patients. In 
fact, the results of network analysis suggest the existence of distinct cognitive deficits in unipolar versus bipolar depression. Indeed, our findings suggest a wider involvement of the various cognitive domains in patients with MDD compared with BD, expressed by a greater density of the unipolar depression network compared to that of bipolar depression. A network is a dynamic entity that highlights the role of each variable with respect to the others, and in clinical terms a greater density means a greater reciprocal influence of the various domains on each other and the simultaneous presence of multiple deficits in the single patient. Thus, our results suggest that patients with unipolar vs. bipolar depression would present a greater predisposition to have a wider range of cognitive deficits, involving multiple domains at the same time. It is important to underline that this result does not represent an estimate of the severity of cognitive impairment: in fact, in patients with unipolar depression, the individual domains could be quantitatively less compromised than in bipolar patients, in which cognitive alterations could be more severe and specific in single isolated domains. Consistently with this result, in a direct comparison study, Taylor Tavares and colleagues (2007) demonstrated a broader spectrum of cognitive impairment in drug-free patients with MDD (including changes in executive functions, set-shifting and working memory) compared to bipolar patients $\mathrm{s}^{32}$. Furthermore, a more recent study indicated that young patients with bipolar II depression had a relatively intact cognitive profile with more isolated and specific deficits compared to patients with unipolar depression ${ }^{33}$. In addition, network metrics provided additional information regarding the role of each cognitive domain. For instance, it is interesting to note that the three domains with the greatest centrality in the unipolar depression network (memory, attention and executive functions) are the same domains for which statistically significant differences emerged, compared to healthy controls, in a meta-analysis of 24 clinical trials (784 total patients) conducted by Rock et al. (2014) ${ }^{60}$. In particular, in the network of unipolar depression, memory is the cognitive domain with the higher centrality. This means that patients with memory impairment more often present other concomitant cognitive changes and that the dysfunction in memory is the cognitive symptom that plays a "gluing" role with the other deficits in unipolar patients. Otherwise, memory does not seem to play such an important role in BD. This is consistent with the findings of a meta-analysis by Xu et al. (2012), in which, during the depressive phase, patients with MDD and BD-I had greater impairment in visual memory than BD-II patients, but no difference emerged in terms of attention, executive functions and information processing speed ${ }^{27}$. Moreover, in a networkanalytic model, where symptoms are considered in their dynamism and the centrality of a symptom is representative of its ability to bring out other symptoms, the fact that memory plays such a central role in MDD may align with the evidence that alterations in memory occur in healthy subjects prior to the development of full-blown depressive symptomatology ${ }^{61}$. In our networks, executive functions emerged among the cognitive domains with the greatest centrality in both unipolar and bipolar depression, though in the latter they represent the cognitive domain with the highest betweenness centrality. In clinical terms, the centrality of executive dysfunctions underlines the importance that alterations in this specific domain play in determining the global cognitive impairment of a bipolar patient. A possible interpretation, in fact, is that a bipolar patient who manifests alterations in executive functions would be more at risk of presenting other cognitive deficits than a patient presenting with isolated memory deficits. In this specific case, the application of the network analysis allows a deeper knowledge of the "dynamic" role of executive functions with respect to classic psychometrics. In fact, if on one hand there were no statistically significant differences between MDD and BD in terms of alteration of executive functions, the network analysis highlighted the most central role played by this cognitive domain in bipolar patients. Indeed, executive dysfunctions have been repeatedly described as one of the main cognitive impairments in $\mathrm{BD}^{62,63}$, with similar patterns in BD-I and BD-II ${ }^{24}$, and they significantly contribute to the disability associated with the disease. Another difference between MDD and BD emerging from the network analysis regarded the more specific role of verbal fluency in bipolar compared to unipolar depression. This is consistent with literature reporting a moderate dysfunction affecting verbal fluency in bipolar patients ${ }^{64}$. In fact, there is evidence that the alteration of verbal fluency appears in the early stages of $\mathrm{BD}$ and worsens with the progression of mood episodes in $\mathrm{BD}^{65}$, but 
not in MDD. Still, from network analysis the relevance of attention in both types of depression emerged. In fact, attention deficit has been repeatedly described in MDD and BD, both during the first episodes of illness ${ }^{29}$ and in remission ${ }^{66}$. Our sample did not present a significant alteration in the domain of attention; however, attention is a moderator of performance in each cognitive test and this could further explain its central role in the networks. Finally, the domains of orientation and visuospatial skills were the most peripheral in both networks. Clinically, therefore, alterations in these domains would emerge only secondarily to alterations in other domains: they would have weak influence on other cognitive functions and would not represent useful markers in order to distinguish bipolar from unipolar depression.

Of note, in this study, we evaluated cognitive performance at two time-points: during acute depression and at remission. With regard to the course of cognitive impairment between these two phases, we found a significant improvement of cognitive function both in unipolar and bipolar patients. Anyway, at remission, the mean MoCA score for MDD patients was just above the threshold of normality, while it was just below it for BD patients, even though such difference was not statistically significant. It is known that in MDD the improvement of cognitive function does not proportionally correspond to the improvement of psychopathological symptoms. For this reason, remitted patients often present with residual cognitive symptoms beyond the single mood episode during euthymia. Moreover, in a previous study by our group ${ }^{67}$, we reported the presence of specific cognitive deficits in adult euthymic patients with BD-I and BD-II. In this perspective, the borderline MoCA scores at time of remission (which was defined on the basis of the HDRS and HARS scores) might depend on the relatively unstable clinical condition patients were at time of evaluation and might reflect the tendency of cognitive symptoms to recover slower than affective symptoms after a MDE.

Reported findings need to be interpreted in light of the following methodological limitations: first of all, the enrollment of a control group would have allowed for the comparison of the cognitive performance of BD and MDD patients with that of healthy controls. Second, there were different treatment patterns between the two groups, due to the naturalistic environment in which the study was conducted. Third, the sample size was relatively limited, in particular with regard to reproducibility of network analysis. For this reasons, further clinical trials on selected drug-free populations are warranted to confirm the evidence emerging from our study.

\section{Conclusion}

In conclusion, during depressive episodes, patients with MDD and BD have similar patterns of cognitive impairment in terms of severity and cognitive domains involved, particularly regarding executive functions and memory. However, the application of the innovative approach of network analysis has allowed to identify some distinctive features between unipolar and bipolar depression: in particular, memory deficits seem to have a more specific role in unipolar depression, whereas executive functions are altered in both subtypes of mood disorders, though with a more prominent role in bipolar depression. Furthermore, attention plays the role of moderator of cognitive function in both MDD and BD, and can therefore be interpreted as a state variable associated with depression itself, rather than a trait variable peculiar of one single mood disorder.

\section{Funding}

The present study was not funded by any institution or company.

\section{Disclosures}


Prof. Dell'Osso has received speaker fees from Lundbeck, Angelini and FB Health. All other Authors declare no conflict of interests.

\section{References}

1. Russo M, Mahon K, Burdick KE. Measuring cognitive function in MDD: emerging assessment tools. Depress Anxiety. 2015;32(4):262-269. doi:10.1002/da.22297.

2. Stordal KI, Lundervold AJ, Egeland J, et al. Impairment across executive functions in recurrent major depression. Nord J Psychiatry. 2004;58(1):41-47. doi:10.1080/08039480310000789.

3. Basso MR, Bornstein RA. Relative memory deficits in recurrent versus first-episode major | depression on a word-list learning task. Neuropsychology. 1999;13(4):557-563.

4. Dickerson FB, Boronow JJ, Stallings CR, Origoni AE, Cole S, Yolken RH. Association between cognitive functioning and employment status of persons with bipolar disorder. Psychiatr Serv. 2004;55(1):54-58. doi:10.1176/appi.ps.55.1.54.

5. Green MF. Cognitive impairment and functional outcome in schizophrenia and bipolar disorder. J Clin Psychiatry. 2006;67(10):e12.

6. Baune BT, Miller R, McAfoose J, Johnson M, Quirk F, Mitchell D. The role of cognitive impairment in general functioning in major depression. Psychiatry Res. 2010;176(2-3):183189. doi:10.1016/j.psychres.2008.12.001.

7. Jaeger J, Berns S, Uzelac S, Davis-Conway S. Neurocognitive deficits and disability in major depressive disorder. Psychiatry Res. 2006;145(1):39-48. doi:10.1016/j.psychres.2005.11.011.

8. Albert U, Brugnoli R, Caraci F, et al. Italian psychiatrists' perception on cognitive symptom $\$$ in major depressive disorder. Int J Psychiatry Clin Pract. 2016;20(1):2-9. doi:10.3109/13651501.2015.1093147.

9. Bortolato B, Miskowiak KW, Köhler CA, et al. Cognitive remission: A novel objective for the treatment of major depression? BMC Med. 2016;14(1):1-18. doi:10.1186/s12916-0160560-3.

10. Farkas M. The vision of recovery today: what it is and what it means for services. World Psychiatry. 2007;6(2):68-74.

11. Biringer E, Mykletun A, Sundet K, Kroken R, Stordal KI, Lund A. A longitudinal analysis of neurocognitive function in unipolar depression. J Clin Exp Neuropsychol. 2007;29(8):879891. doi:10.1080/13803390601147686.

12. Iverson GL, Brooks BL, Langenecker SA, Young AH. Identifying a cognitive impairment | subgroup in adults with mood disorders. J Affect Disord. 2011;132(3):360-367. doi:10.1016/j.jad.2011.03.001.

13. Czepielewski LS, Massuda R, Goi P, et al. Verbal episodic memory along the course of schizophrenia and bipolar disorder: A new perspective. Eur Neuropsychopharmacol. September 2014. doi:10.1016/j.euroneuro.2014.09.006.

14. Maalouf FT, Klein C, Clark L, et al. Impaired sustained attention and executive dysfunction:| bipolar disorder versus depression-specific markers of affective disorders. Neuropsychologia. 2010;48(6):1862-1868. doi:10.1016/j.neuropsychologia.2010.02.015. 
15. Hermens DF, Naismith SL, Redoblado Hodge MA, Scott EM, Hickie IB. Impaired verbal memory in young adults with unipolar and bipolar depression. Early Interv Psychiatry. 2010;4(3):227-233. doi:10.1111/j.1751-7893.2010.00194.x.

16. Wolfe J, Granholm E, Butters N, Saunders E, Janowsky D. Verbal memory deficits associated with major affective disorders: a comparison of unipolar and bipolar patients. $J$ Affect Disord. 1987;13(1):83-92.

17. Robinson LJ, Ferrier IN. Evolution of cognitive impairment in bipolar disorder: a systematic review of cross-sectional evidence. Bipolar Disord. 2006;8(2):103-116. doi:10.1111/j.13995618.2006.00277.x.

18. Bearden CE, Glahn DC, Monkul ES, et al. Patterns of memory impairment in bipolar disorder and unipolar major depression. Psychiatry Res. 2006;142(2-3):139-150. doi:10.1016/j.psychres.2005.08.010.

19. Belleau EL, Phillips ML, Birmaher B, Axelson DA, Ladouceur CD. Aberrant executive attention in unaffected youth at familial risk for mood disorders. $J$ Affect Disord. 2013;147(13):397-400. doi:10.1016/j.jad.2012.08.020.

20. Sweeney JA, Kmiec JA, Kupfer DJ. Neuropsychologic impairments in bipolar and unipolar mood disorders on the CANTAB neurocognitive battery. Biol Psychiatry. 2000;48(7):674684.

21. van der Meere J, Borger N, van Os T. Sustained attention in major unipolar depression. Percept Mot Skills. 2007;104(3 Pt 2):1350-1354. doi:10.2466/pms.104.4.1350-1354.

22. Martinez-Aran A, Penades R, Vieta E, et al. Executive function in patients with remitted bipolar disorder and schizophrenia and its relationship with functional outcome. Psychother Psychosom. 2002;71(1):39-46. doi:10.1159/000049342.

23. Erol A, Kosger F, Putgul G, Ersoy B. Ventral prefrontal executive function impairment as a potential endophenotypic marker for bipolar disorder. Nord J Psychiatry. 2014;68(1):18-23. doi: $10.3109 / 08039488.2012 .756062$.

24. Dickinson T, Becerra R, Coombes J. Executive functioning deficits among adults with Bipolar Disorder (types I and II): A systematic review and meta-analysis. J Affect Disord. 2017;218:407-427. doi:10.1016/j.jad.2017.04.010.

25. Baudic S, Tzortzis C, Barba GD, Traykov L. Executive deficits in elderly patients with major unipolar depression. J Geriatr Psychiatry Neurol. 2004;17(4):195-201. doi:10.1177/0891988704269823.

26. Raucher-Chene D, Achim AM, Kaladjian A, Besche-Richard C. Verbal fluency in bipolar disorders: A systematic review and meta-analysis. J Affect Disord. 2017;207:359-366. doi:10.1016/j.jad.2016.09.039.

27. Xu G, Lin K, Rao D, et al. Neuropsychological performance in bipolar I, bipolar II and unipolar depression patients: A longitudinal, naturalistic study. $J$ Affect Disord. 2012;136(3):328-339. doi:10.1016/j.jad.2011.11.029.

28. Daniel BD, Montali A, Gerra ML, et al. Cognitive impairment and its associations with the path of illness in affective disorders: a comparison between patients with bipolar and unipolar depression in remission. J Psychiatr Pract. 2013;19(4):275-287. doi:10.1097/01.pra.0000432597.79019.e2. 
29. Lee RSC, Hermens DF, Porter MA, Redoblado-Hodge MA. A meta-analysis of cognitive deficits in first-episode Major Depressive Disorder. J Affect Disord. 2012;140(2):113-124. doi:10.1016/j.jad.2011.10.023.

30. Borkowska A, Rybakowski JK. Neuropsychological frontal lobe tests indicate that bipolar depressed patients are more impaired than unipolar. Bipolar Disord. 2001;3(2):88-94.

31. Gruber S, Rathgeber K, Braunig P, Gauggel S. Stability and course of neuropsychological deficits in manic and depressed bipolar patients compared to patients with Major Depression. J Affect Disord. 2007;104(1-3):61-71. doi:10.1016/j.jad.2007.02.011.

32. Taylor Tavares J V, Clark L, Cannon DM, Erickson K, Drevets WC, Sahakian BJ. Distinct profiles of neurocognitive function in unmedicated unipolar depression and bipolar II depression. Biol Psychiatry. 2007;62(8):917-924. doi:10.1016/j.biopsych.2007.05.034.

33. Mak ADP, Lau DTY, Chan AKW, et al. Cognitive Impairment In Treatment-Naive Bipolar II and Unipolar Depression. Sci Rep. 2018;8(1):1905. doi:10.1038/s41598-018-20295-3.

34. Ghaemi N, Sachs GS, Goodwin FK. What is to be done? Controversies in the diagnosis and treatment of manic-depressive illness. World J Biol Psychiatry. 2000;1(2):65-74.

35. Yatham LN. Diagnosis and management of patients with bipolar II disorder. J Clin Psychiatry. 2005;66 Suppl 1:13-17.

36. Nasreddine ZS, Phillips NA, Bedirian V, et al. The Montreal Cognitive Assessment, MoCA: a brief screening tool for mild cognitive impairment. J Am Geriatr Soc. 2005;53(4):695-699. doi:10.1111/j.1532-5415.2005.53221.x.

37. Miskowiak KW, Burdick KE, Martinez-Aran A, et al. Assessing and addressing cognitive impairment in bipolar disorder: the International Society for Bipolar Disorders Targeting Cognition Task Force recommendations for clinicians. Bipolar Disord. January 2018. doi:10.1111/bdi.12595.

38. Burdick KE, Ketter TA, Goldberg JF, Calabrese JR. Assessing cognitive function in bipolar disorder: challenges and recommendations for clinical trial design. J Clin Psychiatry. 2015;76(3):e342-50. doi:10.4088/JCP.14cs09399.

39. Diagnostic and Statistical Manual of Mental Disorders : DSM-5. Fifth edition. Arlington, VA : American Psychiatric Publishing, [2013] (C2013 https://search.library.wisc.edu/catalog/9911111397702121.

40. Hamilton M. A rating scale for depression. J Neurol Neurosurg \&amp; amp; Psychiatry. 1960;23(1):56 LP-62. http://jnnp.bmj.com/content/23/1/56.abstract.

41. Hamilton M. The assessment of anxiety states by rating. Br J Med Psychol. 2018;32(1):5055. doi:10.1111/j.2044-8341.1959.tb00467.x.

42. Folstein MF, Folstein SE, McHugh PR. "Mini-mental state". A practical method for grading the cognitive state of patients for the clinician. J Psychiatr Res. 1975;12(3):189-198.

43. Mitchell AJ. A meta-analysis of the accuracy of the mini-mental state examination in the detection of dementia and mild cognitive impairment. J Psychiatr Res. 2009;43(4):411-431. doi:10.1016/j.jpsychires.2008.04.014.

44. Smith T, Gildeh N, Holmes C. The Montreal Cognitive Assessment: validity and utility in a memory clinic setting. Can J Psychiatry. 2007;52(5):329-332. doi: $10.1177 / 070674370705200508$. 
45. Freitas S, Simoes MR, Alves L, Vicente M, Santana I. Montreal Cognitive Assessment (MoCA): validation study for vascular dementia. J Int Neuropsychol Soc. 2012;18(6):10311040. doi: $10.1017 / \mathrm{S} 135561771200077 \mathrm{X}$.

46. Hoops S, Nazem S, Siderowf AD, et al. Validity of the MoCA and MMSE in the detection of MCI and dementia in Parkinson disease. Neurology. 2009;73(21):1738-1745. doi:10.1212/WNL.0b013e3181c34b47.

47. Freitas S, Simoes MR, Alves L, Duro D, Santana I. Montreal Cognitive Assessment (MoCA): validation study for frontotemporal dementia. J Geriatr Psychiatry Neurol. 2012;25(3):146-154. doi:10.1177/0891988712455235.

48. Yang Z, Abdul Rashid NA, Quek YF, et al. Montreal Cognitive Assessment as a screening instrument for cognitive impairments in schizophrenia. Schizophr Res. March 2018. doi:10.1016/j.schres.2018.03.008.

49. Musso MW, Cohen AS, Auster TL, McGovern JE. Investigation of the Montreal Cognitive Assessment (MoCA) as a cognitive screener in severe mental illness. Psychiatry Res. 2014;220(1-2):664-668. doi:10.1016/j.psychres.2014.07.078.

50. Moirand R, Galvao F, Lecompte M, Poulet E, Haesebaert F, Brunelin J. Usefulness of the Montreal Cognitive Assessment (MoCA) to monitor cognitive impairments in depressed patients receiving electroconvulsive therapy. Psychiatry Res. 2018;259(August 2017):476481. doi:10.1016/j.psychres.2017.11.022.

51. Koenders MA, de Kleijn R, Giltay EJ, Elzinga BM, Spinhoven P, Spijker AT. A Network Approach to Bipolar Symptomatology in Patients with Different Course Types. Wichers M, ed. PLoS One. 2015;10(10):e0141420. doi:10.1371/journal.pone.0141420.

52. Galderisi S, Rucci P, Kirkpatrick B, et al. Interplay Among Psychopathologic Variables, Personal Resources, Context-Related Factors, and Real-life Functioning in Individuals With Schizophrenia: A Network Analysis. JAMA psychiatry. 2018;75(4):396-404. doi:10.1001/jamapsychiatry.2017.4607.

53. Costantini G, Epskamp S, Borsboom D, et al. State of the aRt personality research: A tutorial on network analysis of personality data in R. J Res Pers. 2015;54:13-29. doi:10.1016/j.jrp.2014.07.003.

54. Epskamp S, Cramer AOJ, Waldorp LJ, Schmittmann VD, Borsboom D. qgraph: Network Visualizations of Relationships in Psychometric Data. J Stat Softw. 2012;48(4):1-18.

55. Shannon $\mathrm{P}$, Markiel A, Ozier O, et al. Cytoscape: a software environment for integrated models of biomolecular interaction networks. Genome Res. 2003;13(11):2498-2504. doi:10.1101/gr.1239303.

56. Cramer AOJ, Waldorp LJ, van der Maas HLJ, Borsboom D. Comorbidity: A network perspective. Behav Brain Sci. 2010;33(2-3):137-+. doi:10.1017/s0140525x09991567.

57. Gualtieri CT, Morgan DW. The frequency of cognitive impairment in patients with anxiety, depression, and bipolar disorder: an unaccounted source of variance in clinical trials. J Clin Psychiatry. 2008;69(7):1122-1130.

58. Douglas KM, Gallagher P, Robinson LJ, et al. Prevalence of cognitive impairment in major depression and bipolar disorder. Bipolar Disord. 2018;20(3):260-274. doi:10.1111/bdi.12602. 
59. Trivedi MH, Greer TL. Cognitive dysfunction in unipolar depression: implications for treatment. J Affect Disord. 2014;152-154:19-27. doi:10.1016/j.jad.2013.09.012.

60. Rock PL, Roiser JP, Riedel WJ, Blackwell AD. Cognitive impairment in depression: a systematic review and meta-analysis. Psychol Med. 2014;44(10):2029-2040. doi:10.1017/S0033291713002535.

61. Mannie ZN, Barnes J, Bristow GC, Harmer CJ, Cowen PJ. Memory impairment in young women at increased risk of depression: influence of cortisol and 5-HTT genotype. Psychol Med. 2009;39(5):757-762. doi:10.1017/S0033291708004248.

62. Goswami U, Sharma A, Khastigir U, et al. Neuropsychological dysfunction, soft neurological signs and social disability in euthymic patients with bipolar disorder. $\mathrm{Br} \mathrm{J}$ Psychiatry. 2006;188:366-373. doi:10.1192/bjp.188.4.366.

63. Levy B, Manove E, Weiss RD. Recovery of cognitive functioning in patients with cooccurring bipolar disorder and alcohol dependence during early remission from an acute mood episode. Ann Clin Psychiatry. 2012;24(2):143-154.

64. Bora E, Yucel M, Pantelis C. Cognitive endophenotypes of bipolar disorder: a meta-analysis| of neuropsychological deficits in euthymic patients and their first-degree relatives. $J$ Affect Disord. 2009;113(1-2):1-20. doi:10.1016/j.jad.2008.06.009.

65. Lee RSC, Hermens DF, Scott J, et al. A meta-analysis of neuropsychological functioning in first-episode bipolar disorders. J Psychiatr Res. 2014;57:1-11. doi:10.1016/j.jpsychires.2014.06.019.

66. Clark L, Sahakian BJ. Cognitive neuroscience and brain imaging in bipolar disorder. Dialogues Clin Neurosci. 2008;10(2):153-163.

67. Palazzo MC, Arici C, Cremaschi L, et al. Cognitive Performance in Euthymic Patients with Bipolar Disorder vs Healthy Controls: A Neuropsychological Investigation. Clin Pract Epidemiol Ment Health. 2017;13:71-81. doi:10.2174/1745017901713010071. 


\section{TABLES AND FIGURES}

Table 1. List of inclusion and exclusion criteria

\section{Inclusion criteria}

Male or female gender

$$
\text { Age }>18
$$

Diagnosis of major depressive episode (MDE)

Longitudinal diagnosis of major depressive disorder or bipolar disorder I or II, according to DSM-5 criteria

\section{Exclusion criteria}

Clinically relevant learning or reading disability, dyslexia, illiteracy

Diagnosis of cognitive disorder

History of moderate to severe head injury

Uncontrolled thyroid dysfunction

Recent alcohol or substance abuse

Concomitant therapy with high dose anticholinergics

Concomitant therapy with benzodiazepines at a dosage equal or higher than diazepam $7.5 \mathrm{mg} / \mathrm{day}$

Electroconvulsive therapy within 6 months

Table 2. Demographic and clinical characteristics of the two groups.

\begin{tabular}{|c|c|c|c|}
\hline & MDD & BD & $\mathbf{p}$ \\
\hline Sample size & 72 & 37 & \\
\hline Gender, f\% & $65.3 \%$ & $70.3 \%$ & .600 \\
\hline Age, mean \pm SD & $56.24 \pm 14.51$ & $56.89 \pm 10.02$ & .602 \\
\hline \multicolumn{4}{|l|}{ Marital status, $\%$} \\
\hline Unmarried & $15.3 \%$ & $27.0 \%$ & \multirow[t]{4}{*}{.111} \\
\hline Married & $62.5 \%$ & $59.5 \%$ & \\
\hline Divorced & $11.1 \%$ & $7.3 \%$ & \\
\hline Widow & $11.1 \%$ & $6.2 \%$ & \\
\hline \multicolumn{4}{|l|}{ Education, \% } \\
\hline Primary school & $9.7 \%$ & $8.1 \%$ & \multirow[t]{4}{*}{.904} \\
\hline Secondary school & $6.4 \%$ & $21.6 \%$ & \\
\hline High school & $41.7 \%$ & $48.6 \%$ & \\
\hline University & $22.2 \%$ & $21.6 \%$ & \\
\hline \multicolumn{4}{|c|}{ Employment status, \% } \\
\hline Unemployed & $20.8 \%$ & $27.0 \%$ & \multirow[t]{2}{*}{.744} \\
\hline Employed & $41.7 \%$ & $32.4 \%$ & \\
\hline
\end{tabular}




\begin{tabular}{|c|c|c|c|}
\hline Retired & $37.5 \%$ & $40.5 \%$ & \\
\hline Nationality, italian \% & $93.1 \%$ & $97.3 \%$ & .358 \\
\hline $\begin{array}{l}\text { Family history for } \\
\text { psychiatric disorders, \% } \\
\text { positive }\end{array}$ & $63.9 \%$ & $75.7 \%$ & .212 \\
\hline Age at onset, mean \pm SD & $34.15 \pm 14.94$ & $30.0 \pm 12.89$ & .224 \\
\hline $\begin{array}{l}\text { Duration of untreated illness } \\
\text { (DUI) in months, mean } \pm \text { SD }\end{array}$ & $59.81 \pm 84.43$ & $63.51 \pm 73.33$ & .074 \\
\hline $\begin{array}{l}\text { Duration of illness in years, } \\
\text { mean } \pm \text { SD }\end{array}$ & $22.22 \pm 13.59$ & $26.89 \pm 12.10$ & .087 \\
\hline $\begin{array}{l}\text { Psychiatric comorbidities, } \\
\%\end{array}$ & $27.6 \%$ & $13.5 \%$ & .091 \\
\hline \multicolumn{4}{|c|}{ Comorbidity with personality disorders, $\%$} \\
\hline None & $76.4 \%$ & $73.0 \%$ & \multirow[t]{5}{*}{.463} \\
\hline Cluster A & $1.4 \%$ & $5.4 \%$ & \\
\hline Cluster B & $13.9 \%$ & $10.8 \%$ & \\
\hline Cluster C & $5.6 \%$ & $2.7 \%$ & \\
\hline Not specified & $2.8 \%$ & $8.1 \%$ & \\
\hline $\begin{array}{l}\text { Medical comorbidities, } \% \\
\text { present }\end{array}$ & $48.6 \%$ & $40.5 \%$ & .389 \\
\hline $\begin{array}{l}\text { History of psychosis, } \\
\text { positive } \%\end{array}$ & $19.4 \%$ & $27.0 \%$ & .361 \\
\hline $\begin{array}{l}\text { Hisotry of alcohol abuse, } \\
\text { positive } \%\end{array}$ & $5.6 \%$ & $13.5 \%$ & .153 \\
\hline $\begin{array}{l}\text { History of substance abuse, } \\
\text { positive } \%\end{array}$ & $11.1 \%$ & $2.7 \%$ & .131 \\
\hline $\begin{array}{l}\text { Number of episodes of } \\
\text { illness, mean } \pm \text { SD }\end{array}$ & $3.38 \pm 1.84$ & $5.57 \pm 3.33$ & .001 \\
\hline $\begin{array}{l}\text { Previous hospitalizations, } \\
\text { yes } \%\end{array}$ & $22.2 \%$ & $59.5 \%$ & $<.001$ \\
\hline $\begin{array}{l}\text { Previous suicide attempts, } \\
\text { yes } \%\end{array}$ & $26.4 \%$ & $45.9 \%$ & .030 \\
\hline $\begin{array}{l}\text { Time to remission in days, } \\
m \pm S D\end{array}$ & $77.43 \pm 34.85$ & $84.32 \pm 37.84$ & .382 \\
\hline \multicolumn{4}{|c|}{ Current psychopharmacological treatment } \\
\hline Antidepressants, $\%$ & $93.1 \%$ & $75.7 \%$ & $<.001$ \\
\hline Antipsychotics, $\%$ & $34.7 \%$ & $59.5 \%$ & .019 \\
\hline Mood stabilizers, $\%$ & $4.2 \%$ & $40.5 \%$ & .004 \\
\hline Benzodiazepines, $\%$ & $66.7 \%$ & $56.8 \%$ & .295 \\
\hline \multicolumn{4}{|c|}{ Depressive and anxious symptomatology } \\
\hline $\begin{array}{l}\text { HDRS score during } \\
\text { depression, } \mathrm{m} \pm \mathrm{SD}\end{array}$ & $27.35 \pm 6.05$ & $26.62 \pm 6.45$ & .756 \\
\hline $\begin{array}{l}\text { HARS score during } \\
\text { depression, } \mathrm{m} \pm \mathrm{SD}\end{array}$ & $21.61 \pm 9.062$ & $18.68 \pm 7.32$ & .115 \\
\hline $\begin{array}{l}\text { HDRS score at remission, } \\
\mathrm{m} \pm \mathrm{SD}\end{array}$ & $3.43 \pm 2.64$ & $3.68 \pm 3.15$ & .895 \\
\hline HARS score at remission, & $2.41 \pm 2.58$ & $2.72 \pm 2.66$ & .512 \\
\hline
\end{tabular}


Table 3. Mean scores of MoCA single domains during depression.

\begin{tabular}{|l|ll|l|l|}
\hline \multicolumn{1}{c}{ Maximum values } & \multicolumn{1}{l}{ MDD } & \multicolumn{2}{c}{ BD } & \multicolumn{2}{c}{ p } \\
\hline Memory & 5 & $2.35 \pm 1.71$ & $2.32 \pm 1.58$ & .902 \\
\hline Visuospatial abilities & 4 & $2.81 \pm 1.15$ & $2.89 \pm 1.27$ & .514 \\
\hline Executive functions & 4 & $2.85 \pm 1.08$ & $2.70 \pm 1.31$ & .679 \\
\hline Attention & 6 & $5.40 \pm 0.93$ & $5.30 \pm 1.24$ & .823 \\
\hline Verbal fluency & 6 & $5.01 \pm 1.11$ & $5.11 \pm 0.94$ & .862 \\
\hline Orientation & 6 & $5.81 \pm 0.60$ & $5.68 \pm 0.63$ & .126 \\
\hline MoCA total score & 30 & $24.11 \pm 4.04$ & $23.89 \pm 3.78$ & .709 \\
\hline
\end{tabular}

Formattato: Interlinea: singola

Formattato: Interlinea: singola

Table 4. Matrix of Spearman's rho correlation coefficients between the MoCA domains in MDD and $\mathrm{BD}$ during depression.

\begin{tabular}{|l|l|l|l|l|l|l|}
\hline & MEM & VA & EF & ATT & VF & OR \\
\hline MEM & - & 0.41 & 0.37 & 0.28 & 0.27 & 0.23 \\
\hline VA & -0.07 & - & 0.52 & 0.37 & 0.42 & 0.10 \\
\hline EF & 0.43 & 0.22 & - & 0.40 & 0.63 & 0.29 \\
\hline ATT & 0.54 & 0.06 & 0.46 & - & 0.41 & 0.18 \\
\hline VF & 0.07 & 0.21 & 0.42 & 0.42 & - & 0.24 \\
\hline OR & 0.56 & 0.01 & 0.48 & 0.42 & 0.11 & - \\
\hline
\end{tabular}

BD: coefficients below diagonal; MDD: coefficients above diagonal. MEM: memory; VA: visuospatial abilities; EF: executive functions; ATT: attention; VF: verbal fluency; OR: orientation.

Table 5. Top three domains with the highest betweenness centrality and closeness centrality in the two groups.

\begin{tabular}{|c|c|}
\hline UNIPOLAR DEPRESSION & BIPOLAR DEPRESSION \\
\hline \multicolumn{2}{|l|}{ Betweenness centrality } \\
\hline Memory & Executive functions \\
\hline Executive functions & Attention \\
\hline Attention & Verbal fluency \\
\hline \multicolumn{2}{|l|}{ Closeness centrality } \\
\hline Memory & Executive functions \\
\hline Executive functions & Attention \\
\hline Attention & Memory, visuospatial abilities \\
\hline
\end{tabular}

Formattato: Interlinea: singola 
Figure 1. The unipolar depression network.

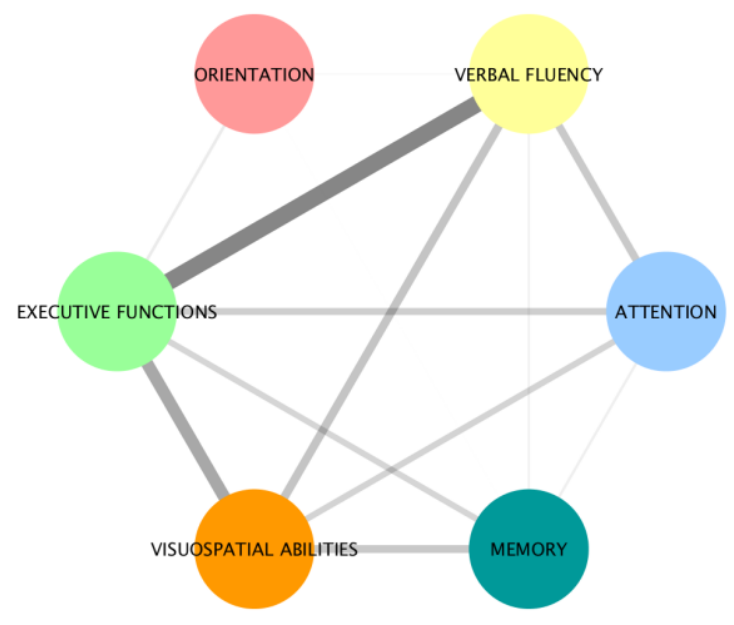

Figure 2. The bipolar depression network.

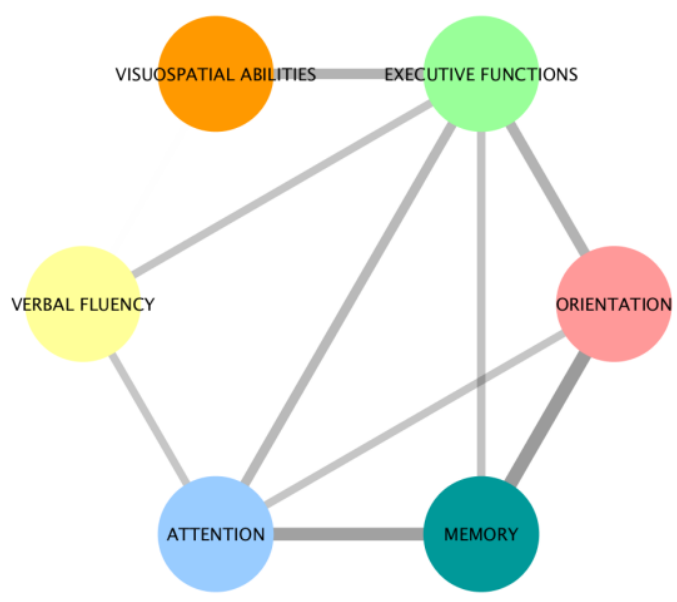

1

Formattato: Interlinea: singola

1

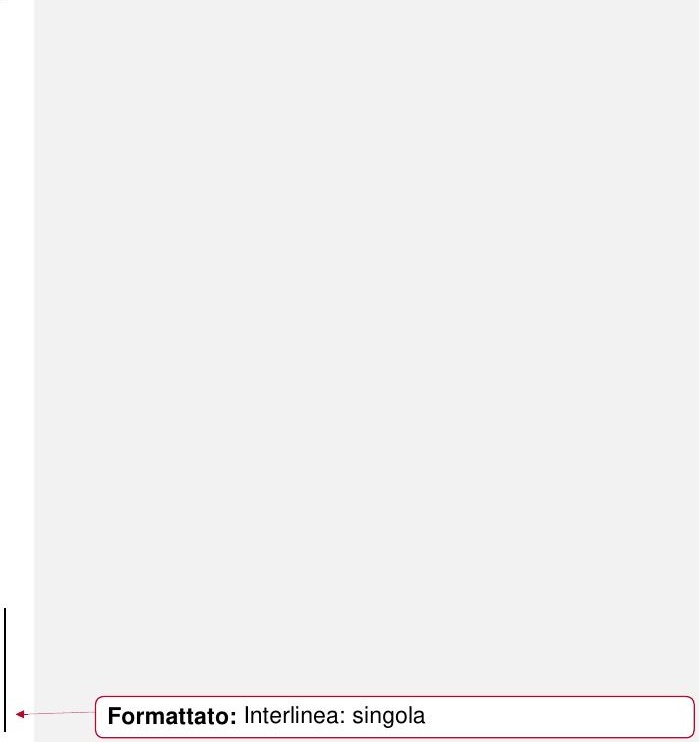

